\title{
The future of psychiatric pharmacogenomics
} "While psychiatry has entered a new area of pharmacogenetics, treatment of
psychiatric patients will always remain personal..."

\section{KEYWORDS: adverse events $\approx$ genetics $\approx$ personalized medicine $\approx$ pharmacogenomics - pharmacology $=$ treatment response}

\section{Clinical need for biomarkers that predict treatment outcome}

Psychiatric disorders are common and have high levels of morbidity and mortality. They strike early in life and are usually lifelong and chronic disorders. Currently, mental illness is ranked as the second in leading source of disease burden in established market economies by the WHO. It is estimated that $10-15 \%$ of the general population will experience clinical depression during their lifetime and $5 \%$ of men and $9 \%$ of women will experience a depressive disorder in a given year (as established by the WHO) [1]. Although there are a variety of pharmacological treatments available, response and tolerability to drugs are highly variable with some patients responding to one treatment but not another. In general, response and remission rates to pharmacological treatment are limited $[2,3]$. There are several potential explanations for these poor drug response rates, including clinical heterogeneity and diagnostic uncertainty, comorbidity, environmental, social and genetic factors. Identification of genetic biomarkers, that can help to predict treatment response of psychotropic medications and aid in the effective treatment of mental illness, would greatly enrich current clinical practice and it is thus of high importance to the field.

\section{Current state of psychiatric pharmacogenomics}

Despite the clear need for better and more effective treatments for psychiatric illnesses, little progress has been made in drug development over the last several decades. Most available drug treatments were initially discovered serendipitously and developed in the 1950s to 1970s. While some refinement has occurred over the last several decades (better tolerability), the overall efficacy and effectiveness of psychotropics has not improved dramatically. More troublesome however is that the mechanism of action of psychotropic drugs and the pathophysiology of most psychiatric disorders remains elusive.

By developing 'personalized' treatment strategies, psychiatric pharmacogenetics has the promise to change this dire picture. Numerous studies have been carried out over the last several years attempting to identify genetic markers that predict drug efficacy and safety. Similar to the field of psychiatric genetics, results have been ambiguous and disappointing and so far there is no clear genetic biomarker that can be used in routine clinical care. To date, there is only one US FDA-approved commercial pharmacogenetic test available (Roche Diagnostic, AmpliChip CYP450) which can be ordered through a few selected commercial and academic laboratories. This test was introduced in 2005 and provides genotypes for the two cytochrome P450 genes; CYP2D6 and CYP2C19. Theoretically, by genotyping patients for variation in these genes, the clinician should be able to predict the metabolizer status of a patient, which might influence medication choice and dosing. There are currently no general guidelines regarding who should get tested; however, several suggestions have been published $[4,5]$.

Nevertheless, new enthusiasm for pharmacogenetics and personalized medicine has been generated over the last several years. Several new findings on gene variants with strong effects prove that pharmacogenetics can be clinically useful. Carbamazepine (CBZ), a drug used to treat seizure disorders, bipolar disorder and chronic pain, has been associated with several severe adverse events, including aplastic anemia, fatal arrhythmias and life-threatening cutaneous disorders. The major histocompatibility complex $H L A-B^{*} 1502$ allele is a strong predictor for CBZ-induced Stevens-Johnson syndrome in Han Chinese individuals [6,7].

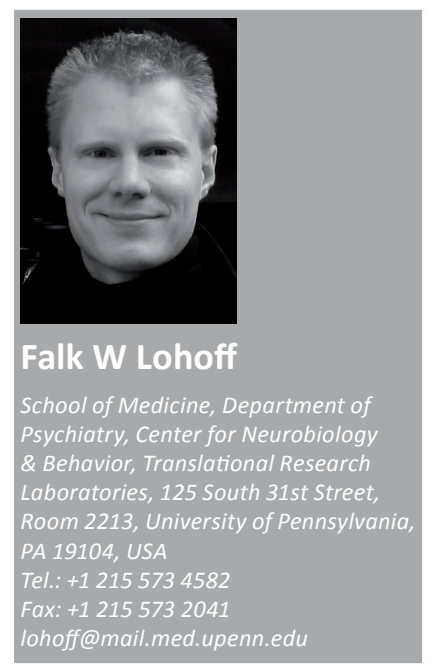

future medicti $_{\text {ns }}$ fsg 
The odds ratio for developing CBZ-induced Stevens-Johnson syndrome if positive for $H L A-B^{*} 1502$ was 2504 with a positive predictive value of $93.6 \%$. As a result of these data, the FDA issued a black box warning in 2007 for CBZ, and in addition, recommended that patients of Asian descent be genotyped for this allele before initiating CBZ therapy. Similar strong associations for genetic biomarkers and severe drug-induced adverse events have been reported in other areas of medicine, such as the association of a specific HLA allele and increased risk for liver injury under fluvoxacillin therapy [8]. With the rapid advance in technology in the field of genetics, it is now reasonable to sequence entire individual patient genomes at relatively low costs. Technological advances in how we study the genome, methylome, transcriptome and proteome will likely identify novel personal markers that predict drug response and adverse events.

\section{Challenges \& future directions}

In order to develop clinically meaningful biomarkers for the clinician, there are several methodological challenges that must be overcome. One of the most fundamental issues in psychiatric pharmacogenetics is that almost all studies so far have used DNA samples from clinical trials that were actually not designed for pharmacogenetic purposes. While it is reasonable to develop hypotheses with post hoc analyses, ultimately prospective pharmacogenetic trials are needed. This poses in particular a challenge for psychiatric drugs, as multiple major pharmaceutical companies have abandoned psychiatric drug development. The field of pharmacogenetics is challenged by weaknesses in both disciplines. Psychiatric drug development has been plagued by negative trials, partly owing to ineffective compounds, but also as a result of clinical heterogeneity, high placebo response rates, weakness in clinical assessments and rating scales and poor study designs. On the genetics side, most studies are underpowered and the selection of genetic markers and gene coverage is insufficient. In order to advance, the field of psychiatric pharmacogenetics needs to develop clear phenotypic definitions, robust outcome measures and comprehensive molecular analysis of biomaterials.

New computational approaches will be necessary to deal with the massive amount of data that will be available and in order to address complex gene-gene interactions and environmental influences. As demonstrated for some
HLA associations with severe adverse events, it is possible to detect meaningful effects in moderate samples sizes. With advances in DNA technology over the past 5 years (e.g., with SNP chips and high-throughput DNA sequencing), the design of well-powered prospective pharmacogenetic trials is feasible. In addition, valid biomarkers will guide and inform research on the biology and mechanism of the underlying disorder and phenotype.

Besides the strong need for methodological changes, the field must be aware and address ethical concerns of personalized medicine. As many medical centers have moved to electronic medical records, there is increased concern regarding privacy and protection of human subjects. In particular, given that platforms such as WikiLeaks are able to obtain highly classified material, it should raise our concern for the privacy of the genetic information of our patients. In addition, no clear regulatory requirements for marketing, intellectual property claims and conduct of pharmacogentic tests exist today. Society and the scientific community need to address the ethical implications of routine biomarker testing, in particular for the more vulnerable population of psychiatric patients.

Although pharmacogenetics in clinical practice is currently limited to 'side effects' and 'metabolism', comprehensive pharmacogenetic profiling will likely soon become a reality. While the technological advancements will offer new possibilities, there are also risks associated with the development of biomarkers (e.g., privacy, limitations of insurance coverage and genetic discrimination). The development of comprehensive pharmacogenetic policies and regulations is critical in order to avoid the misuse of genetic information [9]. While psychiatry has entered a new area of pharmacogenetics, treatment of psychiatric patients will always remain personal. Genetic biomarkers are only one part in the complex constellation of external and internal factors that influence psychopathology and mental health.

Financial \& competing interests disclosure Financial support is gratefully acknowledged from National Institutes of Health grant K08MH080372. The author has no other relevant affliations or financial involvement with any organization or entity with a financial interest in or financial conflict with the subject matter or materials discussed in the manuscript apart from those disclosed.

No writing assistance was utilized in the production of this manuscript. 


\section{Bibliography}

1 Kessler RC, Chiu WT, Demler O, Merikangas KR, Walters EE. Prevalence, severity, and comorbidity of 12 -month DSM-IV disorders in the national comorbidity survey replication. Arch. Gen. Psychiatry 62(6), 617-627 (2005).

2 Trivedi MH, Rush AJ, Wisniewski SR et al. Evaluation of outcomes with citalopram for depression using measurement-based care in STAR*D: implications for clinical practice. Am. J. Psychiatry 163(1), 28-40 (2006).

3 Rush AJ, Trivedi MH, Wisniewski SR et al. Acute and longer-term outcomes in depressed outpatients requiring one or several treatment steps: a STAR*D report. Am. J. Psychiatry 163(11), 1905-1917 (2006).

4 de Leon J, Armstrong SC, Cozza KL. Clinical guidelines for psychiatrists for the use of pharmacogenetic testing for CYP450 2D6 and CYP450 2C19. Psychosomatics 47(1), 75-85 (2006).

5 Mrazek DA. Incorporating pharmacogenetics into clinical practice: reality of a new tool in psychiatry. The context of genetic testing in clinical psychiatric practice. CNS Spectr. 11(Suppl. 3), 3-4 (2006).

6 Chung WH, Hung SI, Hong HS et al. Medical genetics: a marker for StevensJohnson syndrome. Nature 428(6982), 486 (2004).
7 Hung SI, Chung WH, Jee SH et al. Genetic susceptibility to carbamazepine-induced cutaneous adverse drug reactions. Pharmacogenet. Genomics 16(4), 297-306 (2006).

8 Daly AK, Donaldson PT, Bhatnagar P et al. $H L A-B^{*} 5701$ genotype is a major determinant of drug-induced liver injury due to flucloxacillin. Nat. Genet. 41(7), 816-819 (2009).

9 Katsanis SH, Javitt G, Hudson K. Public health. A case study of personalized medicine. Science 320(5872), 53-54 (2008). 